\title{
HIV Pediatric Care Quality in Togo's Health Facilities in 2014
}

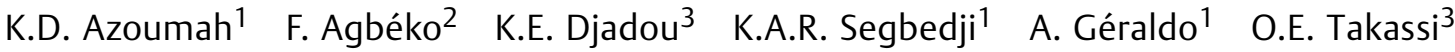 \\ B. Douaguibe ${ }^{4}$ A.D. Agbèrè ${ }^{2} \quad$ Y.D. Atakouma ${ }^{3}$
}

1 Department of Paediatrics, University of Kara, Kara Teaching Hospital, Kara, Togo

2 Department of Paediatrics, University of Lomé, Lomé Commune Regional Hospital, Lomé, Togo

${ }^{3}$ Department of Paediatrics, University of Lomé, Sylvanus Olympio

Teaching Hospital, Lomé, Togo

${ }^{4}$ Department of Obstetrics and Gynecology, University of Lomé, Sylvanus Olympio Teaching Hospital, Lomé, Togo

J Child Sci 2018;8:e11-e17.

Address for correspondence K. D. Azoumah, MD, Department of Paediatrics, University of Kara, Kara Teaching Hospital, Po Box 18, Kara, Togo (e-mail: adkazoum@yahoo.fr; adkazoum@gmail.com).

\begin{abstract}
Keywords

- HIV

- children

- care

- Togo

In 2013, children living with human immunodeficiency virus (HIV)(CLHIV) and on antiretroviral therapy (ART) in Togo accounted for $7.6 \%$ of people living with HIV on ART. Management faces many challenges due to insufficient qualified human resources. This study aimed to assess the availability of care offered to these children in health facilities. This was a retrospective descriptive study on 244 CLHIV (under 15 years) who were on ART and randomly selected in 26 sites providing HIV medical care in Togo from July 22 to September 06 2014. Evaluation forms on children's clinical, biological, and therapeutic parameters were analyzed. Forty percent of CLHIV were between the ages of 5 and 9 years. The average age was 5 years at testing for diagnosis; $49 \%$ of children were in the World Health Organization (WHO) stage III-IV. The sex ratio (male/female $[\mathrm{M} / \mathrm{F}]$ ) was $1: 1$. Almost $71 \%$ of CLHIV were underweight (weight-forage $<-2 Z$ scores Severely underweight : weight-for-age $<-3 Z$ scores). The patient height was not recorded in $81.6 \%$ of the cases. According to the guidelines, the criteria to be on ART were met for $90.2 \%$ of children. The average delay (from diagnosis) to be on ART was 216 days. First-line regimens were mostly zidovudine/lamivudine/ nevirapine (AZT/3TC/NVP) (47.0\%) and stavudine/lamivudine/nevirapine (d4T/3TC) NVP) (38.7\%). Follow-up was characterized by a low rate of achievement of the biological semi-annual evaluation (19.8\%), child compliance for $62.7 \%$, a cotrimoxazole prophylaxis for $70.2 \%$, and tuberculosis screening for $64.8 \%$. The lost-to-follow-up (LFU) proportion was $8.9 \%$. HIV pediatric diagnosis is still an issue in Togo. Early maternal and child healthcare and HIV care are not optimal. Recruitment and capacity building of health professionals, delegation of tasks to paramedics, and innovative motivation processes could improve care for HIV-infected children.
\end{abstract}

\section{Introduction}

According to The Joint United Nations Programme on HIV/ AIDS (UNAIDS), the HIV pandemic has been stabilized in recent years around the world through combined efforts, raising hopes for its total elimination. Africa is one of the regions that has made the most progress in this response. The estimated HIV prevalence in sub-Saharan Africa was $4.7 \%$ (ranging from $0.2 \%$ in Cape Verde to 26.5 in Swaziland). ${ }^{1}$ In Togo, one West African country with a population of received

September 26, 2017 accepted after revision January 23, 2018
DOI https://doi.org/

10.1055/s-0038-1635088. ISSN 2474-5871.
Copyright @ 2018 Georg Thieme Verlag KG Stuttgart · New York
License terms

(c) (i) $\ominus$ (\$) 
$6,600,000$, HIV prevalence was estimated at $2.5 \%$ in 2013 ; children under 15 years constituted $7 \%$ of people living with HIV (PLHIV). ${ }^{1,2}$ Of the 3,275 children living with HIV (CLHIV) registered in healthcare centers, 2,377 (72.6\%) were on antiretroviral therapy (ART), or $11.3 \%$ of all the 21,000 estimated HIV-positive children under 15 years of age in Togo. ${ }^{1,3}$

HIV Prevention Mother-To-Child Transmission (PMTCT) services in 2013 in Togo covered $87.7 \%$ of all health facilities (of which 554/596 were public) while the early infant diagnosis (EID) covered $27.7 \%$ of them. ${ }^{2,4}$ From global recommendations, Togo retained a first ribonucleic acid (RNA) polymerase chain reaction (PCR) for HIV EID at the 6th week's visit and a second for the breastfed child, at 6 weeks after discontinuing any exposure to breast milk. The main EID circuit in Togo is provided by the National AIDS/STI Program, which has contracted with the National Post service to forward dried blood samples (DBS) to the two national reference laboratories (Lomé and Kara). HIV serology (quick tests such as Determine/First Response) was performed in 12-month-old children with confirmation at 18 months when the 12-month test was positive. ${ }^{4-8}$ When CLHIV are put on ART, the first regimen consists of two nucleotide inhibitors and one nonnucleotide inhibitor. The CLHIV are monitored each month for their clinical assessment and for the supply in ART. The LFU is defined as a child who has missed three successive monthly visits. Biological monitoring, consisting at least of a CD4 balance, a hemoglobin level, and an alanine transferase assay, is performed every 6 months. ${ }^{3,5,7}$ Despite progress in HIV care, especially in PMTCT, pediatric coverage for ART was low. ${ }^{1,6}$ Pediatric HIV services are limited, particularly, by the number of insufficient qualified health professionals. ${ }^{2,3}$ This study is aimed to assess the availability of HIV pediatric care in health facilities in Togo.

\section{Methodology}

This study was a descriptive retrospective of HIV-infected children under 15 years in Togo, selected from July 22 to September 06, 2014 in 26 out of 140 sites where PLHIV received medical care. Patients were randomly selected from the ESOPE pediatric monitoring database (Évaluation et Suivi Opérationnel des Programmes ESTHER [Ensemble pour une Solidarité Thérapeutique Hospitalière en Réseau], a French public interest group); their number was retained per site according to the number of patients followed. On the other hand, the choice of the selected health centers took into account the level of healthcare delivery, the type of institution (public, non-governmental organization [NGO], confessional, or private), the rural or urban character, and the PLHIV number followed, according to the geographical locations. In Togo, the healthcare delivery system is organized into a pyramid at three levels: primary, secondary, and tertiary. ${ }^{2,9}$ The primary level, with 1,019 health units, is made up of primary healthcare structures around the health district and comprises two levels: basic health care facilities type I (peripherals units care, medico-social centers, and private care practices) and type II primary care referral facilities (public district hospitals or private health center). The secondary (intermediate) level is represented by the six regional hospitals $(\mathrm{RH})$ and specialized referral hospitals. The tertiary level is constituted by the three teaching hospitals and the specialized health institutions of national scope. The number of health professionals in those health facilities, as of 2013, was 11,140, comprising 459 physicians, 706 senior health technicians (physician assistants) of all categories, 1,374 nurses, 807 midwives, 457 laboratory staff, and 19 clinical psychologists. ${ }^{9}$ The locations of the selected health facilities for this study are provided in - Fig. 1. The institutions selected at the central level for analysis include two of the three reference hospitals in Togo: Sylvanus Olympio teaching hospital at Lomé and Kara teaching hospital. At the intermediate level, a careful choice based on more than 1-year experience in the field of pediatric HIV management and the occurrence of CLHIV accounting for more than $5 \%$ of national CLHIV has led us to retain the RH of Tsévié. At the peripheral level, six districts were selected with CLHIV and without a RH. In the selected districts, in addition to the district hospital, two peripheral care units (Type I or Type II) providing PMTCT services were selected, including a PMTCT site collecting DBS for EID and another PMTCT site not doing so. One of the selected facilities was in a rural area. Each site must have delivered at least one HIV-exposed infant during the year 2013. The confessional health units (covering $11 \%$ of national CLHIV) were included at the rate of one facility in each region: southern Togo and northern Togo. As for the NGO facilities involved in the management of HIV (with $\sim 40 \%$ of CLHIV), two were selected at Lomé and one in northern Togo, as well as a private health facility.

At the time of the survey, the criteria for putting children on ART in Togo, based on revised WHO Pediatric Classification 2006, were ${ }^{5,7}$ : (1) HIV-positive child under 12 months of age with a virological test (positive PCR) regardless of the clinical stage or the percentage of CD4 lymphocytes (confirmed HIV infection), (2) HIV-positive symptomatic child under 18 months of age without a virological test (presumptive diagnosis of severe HIV infection) but with a serological confirmation test at 18 months of age to continue ART, and (3) Child over 18 months of age with confirmed HIV infection, including clinical stage III or IV regardless of the percentage of CD4 lymphocytes, or clinical stage I or II with CD4 lymphocytes $<25 \%$ ( $<1,500$ cells $/ \mathrm{mm}$ ) in children under 12 months, CD4 lymphocytes $<20 \%(<750$ cells $/ \mathrm{mm}$ ) in children aged 12 to 35 months, CD4 lymphocytes $<20 \%$ $(<350$ cells $/ \mathrm{mm})$ in children over 36 months.

CLHIV who have fulfilled the conditions to be put on ART should receive antiretroviral (ARVs) as soon as possible. Any CLHIV under 5 years and at clinical stage III-IV regardless of age needed a cotrimoxazole chemoprophylaxis. A tuberculosis screening was performed in those children according to a syndromic approach taking into account the presence of TB symptoms (cough and chest pain). This is supported by the acid-fast bacilli (AFB) search in the sputum or in the gastric fluid and a chest X-ray test.

This study was conducted as part of a general retrospective analysis of pediatric care management in CLHIV 


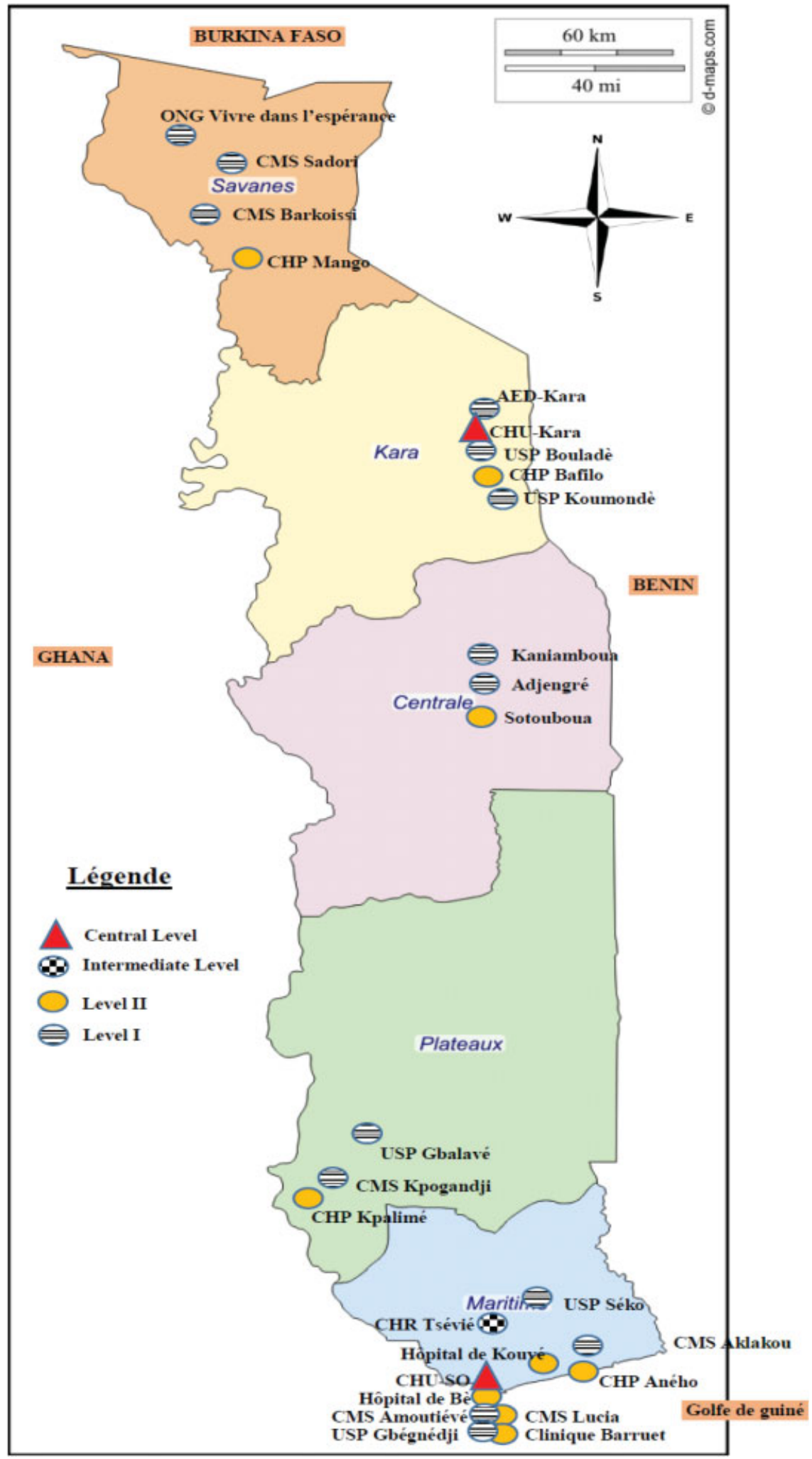

Fig. 1 Togo's maps with the 26 selected health facilities locations.

that was approved by the national bioethics committee. Under this ethics clearance, the consent of guardians was not required for retrospective analysis of routine clinical care data. The data collection was provided by a team of 12 trained health professionals (four pediatricians, two general practitioners, five senior health technicians, and one statistician) after training. The administrated questionnaires were designed to assess the clinical (age, sex, anthropometry, WHO clinical stage, and diagnosis), biological (serology, PCR, and CD4 percentage), and therapeutic (ART initiation 
and follow-up) parameters of the CLHIV as well as to identify the resources and activities of the care centers. Quantitative analysis, performed with Stata, was focused on key indicators such as anthropometric, diagnostic, therapeutic, and evolutionary parameters. The Tanahashi method was also used to determine the level of the various services offered to children.

\section{Results}

Outside of the unavailable data from Sylvanus Olympio Teaching Hospital, the biggest center of PLHIV care management, CLHIV on ART in selected sites represented $9.4 \%$ of on ART PLHIV or 814 CLHIV. A sample of 244 children was selected.

\section{HIV Diagnosis and Antiretroviral Therapy in Surveyed Children}

The average age at diagnosis for CLHIV was 5 years. The most represented age groups were those of 5 to 9 years (40\%) and those of 2 to 4 years (25\%). Infants under 2 years accounted for $21 \%$ of the total children while adolescents aged 10 to 15 years accounted for $14 \%$. Male children made up 53.3\% (130/244) of the sample.

The diagnosis of HIV infection was done mainly by serological testing after 18 months (90.5\%), or to a lesser extent by presumptive diagnosis before 18 months $(5.4 \%)$ or PCR (4.1\%). The initial clinical evaluation $(n=213)$ revealed that most children were in stage III (35\%) or IV (14\%) according to the WHO classification. Twenty-six percent of them were in stage II, and $25 \%$ were asymptomatic. Seventy-one percent of children were underweight (weight-for-age $<-2 Z$ scores), of which $41 \%$ were severely underweight. The patient height was not recorded in $82 \%$ of the cases, and this was not enough to appreciate nutritional and growth status disorders. Biological evaluation (in 198 children) revealed a mean rate of CD4 at 607. The interpretation of this CD4 level by age (reference to the WHO standard) showed $77.3 \%$ of patients with immunosuppression, of which $35 \%$ were severe (70 cases) and $42 \%$ were moderate ( 82 cases), correlating with advanced clinical stages. In $22.8 \%$ of the cases CD4 was normal.

ART initiation occurred at a mean age of 5.4 years. In $90 \%$ of the cases (220 children), guidelines were compliant with ART criteria as defined by the WHO in 2010 and the national protocol: CD4 inferior to $25 \%$ and WHO stages III-IV made up the most common criteria used to initiate ART ( - Table $\mathbf{1}$ ). The average delay (from diagnosis) to start an ART was 216 days. - Table 2 summarizes the different ART received by children. The first-line regimen was correct in 96\% (208/217) of patients older than 3 years, mainly constituted by zidovudine/lamivudine/nevirapine (AZT/3TC/NVP) (47.0\%) and stavudine/lamivudine/nevirapine (d4T/3TC/NVP) (38.7\%) in accordance with national guidelines in progress. Under the age of 2 years, 6/14 children were on the protease inhibitorbased regimen, and $7 / 14$ were NVP based.

More than half of the HIV-infected children were followed up in public health facilities (55.5\%), one third in NGO
Table 1 Distribution of children according to ART criteria

\begin{tabular}{|l|l|l|}
\hline ART criteria & $\begin{array}{l}\text { Number } \\
(\boldsymbol{n}=\mathbf{2 2 0})\end{array}$ & $\begin{array}{l}\text { Percentage } \\
(\mathbf{\%})\end{array}$ \\
\hline CD4 counts $<25 \%$ & 123 & 55.9 \\
\hline WHO Stages III and IV & 106 & 48.2 \\
\hline Positive PCR & 08 & 03.6 \\
\hline $\begin{array}{l}\text { Children living with HIV } \\
<18 \text { mo old }\end{array}$ & 03 & 01.4 \\
\hline
\end{tabular}

Abbreviations: ART, antiretroviral therapy; HIV, human immunodeficiency virus; PCR, polymerase chain reaction; WHO, World Health Organization.

Note: Certain criteria can be combined in children to start an ART.

(30.6\%), and $13.6 \%$ in confessional facilities. Children in the private sector accounted for $0.3 \%$ of infected children. In CLHIV follow-up, the absence of the height measurement ( $85.2 \%$ or 208 cases), that of the weight ( $20.8 \%$ or 51 cases) and a low rate of biannual biological follow-up (19.8\% or 45 cases) were recorded in the files. Two-thirds of the children (62.7\% or 146 cases) were ARV compliant. The prevention of opportunistic infections was done using cotrimoxazole in $70.2 \%$ (170 cases) and tuberculosis (TB) screening in $64.8 \%$ (153 cases). The LFU (out of sight for 3 months) proportion was $8.9 \%$ (19 cases). Survival at 12 months on ART of children surveyed was $83.2 \%$, and that of those at 24 months was $61 \%$.

\section{Providing of Pediatric Care at the Sites}

Concerning the level of availability of maternal, newborn, and child health (MNCH), PMTCT and pediatric ART services in the different sites surveyed ( - Table 3 ), provider-initiated HIV testing and counseling (PITC) (27\%), psychosocial monitoring (42\%), and antiretroviral drugs dispensing (50\%) were the least available pediatric care management. Infants'

Table 2 Distribution of children by primary ART regimen received

\begin{tabular}{|l|l|l|}
\hline First-line regimen & $\begin{array}{l}\text { Number } \\
(\boldsymbol{n}=\mathbf{2 1 7})\end{array}$ & $\begin{array}{l}\text { Percentage } \\
(\%)\end{array}$ \\
\hline 3TC/AZT/NVP & 102 & 47.0 \\
\hline $\mathrm{d} 4 \mathrm{~T} / 3 \mathrm{TC} / \mathrm{NVP}$ (before 2012) & 84 & 38.7 \\
\hline $\mathrm{ABC/3TC/LOP/R}$ & 7 & 3.2 \\
\hline $\mathrm{AZT/3TC/LOP/R}$ & 6 & 2.8 \\
\hline ABC/3TC/NVP & 4 & 1.8 \\
\hline AZT/3TC/EFV & 3 & 1.4 \\
\hline TDF/3TC/EFV & 3 & 1.4 \\
\hline ABC/3TC/EFV & 2 & 0.9 \\
\hline Other combinations & 6 & 2.8 \\
\hline
\end{tabular}

Abbreviations: $A B C$, abacavir; ART, antiretroviral therapy; $A Z T$, zidovudine; d4T, stavudine; EFV, efavirenz; LOP/R, lopinavir/ritonavir; NVP, nevirapine; 3TC, lamivudine; TDF, tenofovir. 
Table 3 Level of availability of MNCH services and pediatric HIV care by level of healthcare delivery

\begin{tabular}{|c|c|c|c|c|c|}
\hline \multirow{2}{*}{$\begin{array}{l}\text { Level of health care } \\
\text { delivery }\end{array}$} & \multirow{2}{*}{$\begin{array}{l}\text { Central and } \\
\text { intermediate } \\
\text { level }(n=3)\end{array}$} & \multicolumn{3}{|c|}{ Primary level $(n=23)$} & \multirow{2}{*}{$\begin{array}{l}\text { Total of facilities } \\
(n=26)\end{array}$} \\
\hline & & $\begin{array}{l}\text { Confessional } \\
\text { and NGO } \\
\text { institutions } \\
(n=5)\end{array}$ & $\begin{array}{l}\text { Peripheral } \\
\text { level } 1 \\
(n=12)\end{array}$ & $\begin{array}{l}\text { Peripheral } \\
\text { level } 2 \\
(n=6)\end{array}$ & \\
\hline \multicolumn{6}{|c|}{ MNCH services and pediatric HIV care } \\
\hline $\begin{array}{l}\text { Mother-baby follow-up } \\
\text { in PMTCT }\end{array}$ & $3(100 \%)$ & $3(60 \%)$ & $12(100 \%)$ & $5(83 \%)$ & $23(88 \%)$ \\
\hline EID & $3(100 \%)$ & $2(40 \%)$ & $12(100 \%)$ & $3(50 \%)$ & $17(65 \%)$ \\
\hline PITC & $3(100 \%)$ & $3(60 \%)$ & $6(50 \%)$ & $1(17 \%)$ & $7(27 \%)$ \\
\hline ART & $3(100 \%)$ & $5(100 \%)$ & $12(100 \%)$ & $2(33 \%)$ & $17(65 \%)$ \\
\hline $\begin{array}{l}\text { Antiretroviral drugs } \\
\text { dispensing }\end{array}$ & $3(100 \%)$ & $4(80 \%)$ & $12(100 \%)$ & $0(0 \%)$ & $13(50 \%)$ \\
\hline Infants' consultation & $3(100 \%)$ & $5(100 \%)$ & $12(100 \%)$ & $6(100 \%)$ & $26(100 \%)$ \\
\hline Vaccination & $3(100 \%)$ & $2(40 \%)$ & $12(100 \%)$ & $6(100 \%)$ & $23(88 \%)$ \\
\hline Nutrition & $3(100 \%)$ & $3(60 \%)$ & $12(100 \%)$ & $4(67 \%)$ & $19(73 \%)$ \\
\hline Psychosocial monitoring & $3(100 \%)$ & $4(80 \%)$ & $6(50 \%)$ & $1(17 \%)$ & $11(42 \%)$ \\
\hline
\end{tabular}

Abbreviations: ART, antiretroviral therapy; EID, early infant diagnosis; HIV, human immunodeficiency virus; MNCH, maternal, newborn and child health; NGO, non-government organization; PITC, provider-initiated HIV testing and counseling; PMTCT, prevention mother-to-child transmission.

consultation (100\%), follow-up of HIV-infected mothers and their children (88\%), vaccination (88\%), and nutritional management $(73 \%)$ were the most commonly available services across all sites. MNCH and pediatric HIV services were complete at the central and intermediate level facilities (100\% for each of the services), lower and incomplete at the peripheral level and in the private health facilities. At the peripheral level 1, PITC and psychosocial care only existed in $50 \%$ of the cases. Also, only one of the six district hospitals had a psychologist, and two of them had neither a psychologist nor a counselor. At the peripheral level 2, the most available services were infants' consultation (100\%), immunization (100\%), and PMTCT (83\%). In half of the cases, EID and nutrition were not available. There was little ART (25\%), and PITC was almost absent (8\%). According to the private sector, MNCH was poorly developed: $\mathrm{PMTCT}$ at $60 \%$, vaccination at $40 \%$, management of malnutrition at $60 \%$, and EID by DBS at $40 \%$. Confessional health facilities in this sector had a comprehensive package of services (100\%), which was better than that at NGO and private centers.

The HIV-infected children were monitored at the main hospitals by a pediatrician, a general practitioner, or a senior health technician (physician assistant). At the peripheral care unit, this monitoring did not exist or was limited at a control and promotion of the growth and immunization visits. With NGO, the follow-up with the infected child was performed concomitantly with the hospital follow-up because there is neither a vaccination unit nor an early diagnosis.

\section{Discussion}

The average age at HIV diagnosis in CLHIV was 5 years in the study conducted. The initiation of ART began in an average delay of 216 days (31 weeks). HIV diagnosis and ART initia- tion were delayed in this study. The same findings were made by other sub-Saharan researchers. ${ }^{10-12}$ The diagnosis was often made very late, around 5 years, which explained the advanced clinical stages (49\% in Stages III-IV) as well as moderate or severe immunosuppression cases (this series:77\%, Nigeria:78.4\%, and Tanzania:48\%). ${ }^{10,12}$ This situation is somewhat better in Malawi, where from October 2004 to October 2010, the median admission age was 24 months (interquartile range [IQR]: $12-62$ ). ${ }^{13}$ If opportunistic affections could not be well reported in this study, malnutrition, pneumonia, and malaria were the most common primary admission diagnoses. ${ }^{13}$ In CLHIV (age 5-14 years) who had not yet started the ART and were enrolled in four HIV subSaharan African programs, advanced HIV disease at presentation (low body mass index, stage 3 or 4, low CD4 count, or tuberculosis diagnosis) was associated with increased mortality and LFU. ${ }^{14}$ In Nigeria, the average time to initiate ART was 26 weeks ( $42 \%$ of the children had an initiation delay of 12 weeks), and $81.5 \%$ of CLHIV were on AZT/3TC/NVP combination therapy. ${ }^{10}$ In this study, the ART was based on AZT/ $3 T C / N V P(47.0 \%)$ and $\mathrm{d} 4 \mathrm{~T} / 3 \mathrm{TC} / \mathrm{NVP}$ (38.7\%). These regimens are administered based on countries' resources.

Early diagnosis of HIV and related diseases and their treatment, in the study area, face several pitfalls, which need to be improved: disruption of inputs data, geographical accessibility, managerial and organizational problems, inadequate human resources, and training problems. $3,6,10,15$ To avoid rapid progression in CLHIV and death, this diagnosis and treatment must be done early, and all HIV-infected children must be initiated on ARVs irrespective of their clinical or laboratory status. Late presentation and delays in initiating ART among eligible children were the basis for a large incidence of patient losses during pre-ART follow-up in sub-Saharan Africa. ${ }^{14}$ Strategies must be developed to 
shorten this delay, such as delegation of tasks and training of paramedic caregivers to cope with the insufficiency of physicians in health centers. ARVs dispensation must be introduced on all sites offering care to the PLHIV; patients sometimes moved to get ARVs in accredited sites. Only $56 \%$ of 140 sites where PLHIV receive medical care ( 70 sites) in Togo dispensed ARVs at the time of the study. This ART was stabilized and improved by the WHO 2015 recommendations stipulating $\mathrm{ABC} / 3 \mathrm{TC} / \mathrm{LOP} / \mathrm{R}$ as the first-line regimen. ${ }^{16}$

The results also indicate an insufficient quality of care for followed up ART CLHIV. There were missing data in clinical monitoring (anthropometric, clinical signs, and diagnosis) and the biological checking was performed in only $19.8 \%$ of cases. Concerning cotrimoxazole, chemoprophylaxis (performed in $70.2 \%$ ), stock-outs, non-control, or omission of indications by prescribers were the main reasons for this non-optimal prevention. As for TB research, it was performed in two-thirds (64.8\%) of cases, while it was systematically recommended. TB prevalence in Togo was 104 for 1,00,000 inhabitants in 2012. ${ }^{17}$ An emphasis should be placed on training and sensitization of prescribers on TB/HIV co-infection and other HIV-related opportunistic diseases. Monitoring of the CLHIV on ART is important to ensure better follow-up and compliance to reduce the occurrence of infections. In Togo, in 2009, followup of CLHIV on ART in rural areas showed a decrease in severe acute malnutrition (56\% after 3 months of follow-up to $25 \%$ at 12 months) and an increase in the number of CD4 in $60 \%$ of children after 12 months of treatment. ${ }^{11}$

A similar study to this one in Nigeria (2012) showed a follow-up failure in $35 \%$ and a mean duration of child followup of 24.4 months. ${ }^{10}$ Children who were lost sight of during follow-up in these series accounted for $8.9 \%$ (over 3 months), a better proportion than reported in Nigeria and Zambia (respectively, $19.1 \%$ and $14 \%$ over 2 years). ${ }^{10,15}$ Moderateto-severe immunosuppression, age less than 2 years, and a delay of ART initiation greater than 12 weeks were significantly associated with loss to follow-up. ${ }^{10,15}$ The mortality rate in this study at 12 months ART follow-up was $16.8 \%$. This mortality rate was $4.2 \%$ in Nigeria and $5 \%$ in Zambia during follow-ups. Follow-up failure requires qualitative and quantitative reinforcement of the care staff, a good psychosocial accompaniment for children, and a minimum standard of equipment and conditions for care centers to provide a betterquality care.

This descriptive and retrospective evaluation was limited by the deficiencies in the data quality collected due to inadequacies in the patient monitoring system and health information system. However, it gives us important information about the level of care and services offered to CLHIV in our facilities to improve the quality of this care. With regard to the availability of $\mathrm{MNCH}$, provider-initiated HIV testing and counseling (PITC) (27\%), psychosocial monitoring (42\%), and antiretroviral drugs dispensing (50\%) were the least available pediatric care management, followed by EID (65\%). Apart from the PITC strategy, providers have not yet integrated the proposal for screening in childcare, a screening only performed in case of presumption. Furthermore, the proportion of health institutions practicing PITC in Togo was low (20 pilot centers in 2013 , i.e., $2 \%$ ). The exercise of this approach stopped at main hospitals where the complete care package was available. Medical care sites were mainly urban (85\%) consisting of public health institutions (50\%), NGO (25\%), and confessional institutions (11\%). ${ }^{17}$ The proportion of HIV screening tests given in Togo was very low $(2.7 \%)$ among children under15 years at voluntary screening centers in 2014 (PITC strategy performed in 5\% of children at pilot centers showed $221 \mathrm{HIV}$-infected children or a rate of 6\%). ${ }^{17}$ An obvious gap in missed opportunity for EID of HIV (performed in 13\%) was also observed in comparison with Pentavalent 1 vaccination (performed in $89 \%)^{4,17}$ showing the need to reinforce early diagnosis and PITC to scale up a more rigorous identification of these children. The Togolese context is marked by an insufficiency of skilled and trained health professionals: 1 doctor for 16,670 inhabitants and 1 nurse per 5,000 inhabitants. ${ }^{2}$ Only doctors and senior health technicians were authorized by the national HIV/AIDS policy to care for CLHIV on ART. The follow-up of CLHIV was almost non-existent in peripheral level 1 (peripheral unit care and medico-social centers) where care offers are limited to vaccination and control of population growth. The insufficiency of human resources (both in quality and quantity) in health centers are also the basis of the non-optimal level of psychosocial monitoring and ARVs dispensing. In this study, psychologists (in 8 sites out of 26) and counselors, key stakeholders who can assist in therapeutic education and improve the functioning of the circuit, are very insufficient or non-existent, or recruited temporarily for one-time projects. To reach better integrative HIV pediatric care in our institutions, the role of care managers must be considered. In this management in Togo, focal points were selected among care providers in health districts and hospitals to ensure input management and follow-up. In Zambia, in 2011, barriers to the provision of quality pediatric HIV treatment included challenges in diagnosing treatment failure, human resources constraints, lack of caregiver involvement, lack of disclosure, limited adolescent-specific care, dysfunctional laboratory systems, and inadequate data management systems. ${ }^{15}$ These findings reinforce the need for a comprehensive care package. In 2012, implementation of a comprehensive package HIV care in rural Tanzanian clinic resulted in an increased number of mothers and children diagnosed and linked into care, a higher detection of children with AIDS, universal treatment coverage, lower loss to follow-up, and an early mother-to-child transmission rate below elimination limit. ${ }^{12}$ A successful implementation of a care delivery value chain as a strategy to optimize care delivery and inform quality improvement efforts was also reported in a NGO in northern Togo. ${ }^{18}$ These experiences must inspire public pediatric HIV/ AIDS care management to improve the quality of pediatric HIV care.

\section{Conclusion}

HIV diagnosis and therapy in children are still delayed. Despite significant efforts noted in Togo, the monitoring of CLHIV needs to be improved. Maternal, neonatal, and child 
health care and HIV care are not optimal, partially integrated into main public health centers and more limited in urban areas. Integrating HIV pediatric care is a necessity for better survival of CLHIV. This will inevitably require strengthening the capacity of health professionals, delegating tasks to other health workers, monitoring the integration of various maternal and child care, and performance-based funding to improve the comfort and life expectancy of these children in resource-limited settings.

\section{Conflict of Interest}

Dr. Azoumah reports non-financial support from The United Nations Children's Emergency Fund (UNICEF) and from the Program National de lutte contre le SIDA et les IST du Togo (PNLS-Togo) during the conduct of the study.

\section{Acknowledgments}

This study was funded by the United Nations Children's Fund (UNICEF) Togo and the Program National de Lutte contre le SIDA/IST du Togo (Togo National AIDS/STI Program).

\section{References}

1 UNAIDS. Global Report: UNAIDS Report on the Global AIDS Epidemic 2013. Geneva, Switzerland: UNAIDS; 2013. Available at: http://www.unaids.org/sites/default/files/media_asset/UNAIDS_Global_Report_2013_en_1.pdf

2 Ministère de la Planification du Développement et de l'Aménagement du Territoire (MPDAT), Ministère de la Santé (MS) et ICF International. Togo Enquête Démographique et de Santé 20132014. Rockville, Maryland, USA : MPDAT, MS et ICF International 2015. Available at: https://dhsprogram.com/pubs/pdf/FR301/FR301. pdf

3 Ministère de la santé et de la protection sociale. Rapport annuel2015 des activités du PNLS-IST. Lomé, Programme National de Lutte contre le Sida et les infections sexuellement transmissibles - Ministère de la Santé du Togo; 2015. Available at: http://www.pnls.tg/rapports/RAPPORTANNUELPNLS2015.pdf

4 Azoumah KD, Agbeko F, Segbedji KAR, et al. Assessment of the Early Infant Diagnosis of HIV Infection in Togo in 2014. J AIDS Clin Res 2017;8(05). Doi: 10.4172/2155-6113.1000695

5 Grandir. Guide de prise en charge de l'infection à VIH chez l'enfant. Manuels pour les apprenants impliqués dans la prise en charge pédiatrique du VIH. Sidaction Edition 2010. Available at: https://www.medbox.org/guide-de-prise-en-charge-de-linfection-a-vih-chez-lenfant/download.pdf
6 Bouraima M, Salou M, Tchounga BK, et al. Accessibility of early infant diagnosis of HIV infection in Lome (Togo). Arch Pediatr. 2014;21(10):1093-1100. doi: 10.1016/j.arcped.2014.07.006

7 Sidaction. Guide de prise en charge de l'infection à VIH chez l'enfant et de l'adolescent. Grandir. Paris, Sidaction; 2010. Available at: https://www.yumpu.com/fr/document/view/17249965/ guide-de-prise-en-charge-de-linfection-a-vih-chez-l'enfantetdel'adolescent/2010.pdf

8 World Health Organization. WHO recommendations on the diagnosis of HIV infection in infants and children. WHO; 2013. Available at: http://www.who.int/hiv/pub/paediatric/diagnosis/ en/index.html. Accessed July 13, 2013

9 Ministère de la santé. Principaux indicateurs de santé au Togo 2014. Lomé, Ministère de la santé et de la protection sociale; 2015. Available at: http://sante.gouv.tg/sites/default/files/documents/ principaux_indicateurs_2014_du_16-12-15_vf.pdf

10 Greeson D, Ojikutu B, Kolapo U, Higgins-Biddle M, Cabral H, Cooper E. Rapid Assessment of Pediatric HIV Treatment in Nigeria. Arlington, VA: USAID's AIDS Support and Technical Assistance Resources, AIDSTAR-One, Task Order 1; 2013 Available at: http:// www.aidstar-one.com/focus_areas/treatment/resources/report/ pediatric_tx_nigeria

11 Djadou KE, Azoumah DR, Saka B, et al. Follow-up of HIV infected children receiving antiretroviral therapy in a rural area of Togo. Med Sante Trop 2012;22(03):283-286

12 Gamell A, Glass TR, Luwanda LB, et al; KIULARCO Study Group. Implementation and operational research: an integrated and comprehensive service delivery model to improve pediatric and maternal HIV care in rural Africa. J Acquir Immune Defic Syndr 2016;73(05):e67-e75

13 Nosek CA, Buck WC, Caviness AC, et al. Hospital admissions from a pediatric HIV care and treatment program in Malawi. BMC Pediatr 2016;16:22

14 Bastard M, Poulet E, Nicolay N, Szumilin E, Balkan S, PujadesRodriguez M. Pediatric access and continuity of HIV care before the start of antiretroviral therapy in sub-Saharan Africa. Pediatr Infect Dis J 2016;35(09):981-986

15 Greeson D, Ojikutu B, Pitorak H, Cabral H, Cooper E. Rapid Assessment of Pediatric HIV Treatment in Zambia. Arlington, VA: USAID's AIDS Support and Technical Assistance Resources; 2012, AIDSTAR-One, Task Order 1

16 World Health Organization. Recommendations on the diagnosis of HIV infection in infants and children. WHO; 2015. Available at: http://www.who.int/entity/hiv/pub/arv/chapter2.pdf?ua

17 Ministère de la santé. Rapport annuel PNLS-IST 2014. Lomé, Programme National de Lutte contre le Sida et les infections sexuellement transmissibles du Togo-Ministère de la santé 2014. Available at: http://www.pnls.tg/rapports/RapportannuelPNLS/ 2014.pdf

18 Fiori K Jr, Schechter J, Dey M, et al. Closing the delivery gaps in pediatric HIV care in Togo, West Africa: using the care delivery value chain framework to direct quality improvement. AIDS Care 2016;28(Suppl 2):29-33 